\title{
Practical energy-saving in 3G femtocells
}

\author{
Ivaylo Haratcherev, Alberto Conte \\ Networking Technology Domain \\ Alcatel-Lucent Bell Labs France \\ Centre de Villarceaux, 91620 Nozay, France
}

\begin{abstract}
Cell stand-by is widely considered today as one of the most promising techniques to reduce energy consumption of cellular base stations. The majority of existing studies assume that cells can simply enter standby mode, but very few measurements and practical studies exist. In this paper we present a practical implementation of standby mode on a commercial 3G femtocell, the lessons learned and the results obtained.
\end{abstract}

Keywords: energy-saving, energy-efficiency, femto base station, femtocell, 3G, implementation, measurements

\section{INTRODUCTION}

The energy efficiency of cellular networks is a well-known and largely studied problem today. Recent analysis of the power consumption of cellular networks shows that most of the energy is consumed by the base stations (BS), with a ratio close to $80 \%$ [1]. The improvement of BS efficiency is therefore crucial for a sustainable evolution of cellular networks.

Cell stand-by is considered as one of the most promising techniques to reduce energy consumption of cellular base stations, especially in those covering small area like femtocells [2]. It exploits the fact that during low-load periods some of the BS become idle and can enter a low-consumption mode. A lot of literature exists on the subject, from scenario performance (savings) evaluation, to algorithms for selecting the best cells to be kept active. Unfortunately, quite insufficient information exists on practical implementation of such low-power modes. It is our belief that realistic measurements on achievable gains and wake-up delays are necessary to validate algorithms and performance estimations. For example, solutions assuming that cell wake-up is instantaneous are not realistic.

This paper presents the outcomes of an implementation of a standby mode (with its companion wake-up procedure) on a commercial $3 \mathrm{G}$ femtocell. We show the implementation options and their practical limitations, explain the rationale behind our choices (e.g. which components to turn-off, how to wake-up) and report the measurement results on both energy savings and state transition delays.

We conclude the paper with a brief description of possible next steps.

\section{RELATED WORK}

Theoretical aspects of energy-saving in some forms of telecommunication equipment have been studied in detail for at least a decade now. Examples are wireless sensor networks and mobile handsets where energy efficiency (maximizing battery life) is an important issue.

But just in the last five years increasing concerns about energy consumption in communication networks provoked an attention shift to energy saving in core and access networks. Approaches vary from strategies on macro (network system) level, to studies on device (network element) level, to improvements on subsystem or component level (e.g. advances in power amplifier design). A typical macro approach is [7], where Chiaraviglio et al. showed that a large amount of energy can be saved if an optimal dynamic radio coverage planning is used instead of a static one for an UMTS access network. In particular, they switched off some cells in urban areas during low-traffic periods. An example of a device-level study is [8], where authors discuss sleep modes for the base stations (BS) of a cellular access network and focus on the design of BS sleep and wake-up transients. The conclusion is that BS sleep modes can be very effective in reducing energy consumption and might become pervasive in the wireless networks of the future. Research on component level is also actively going on, for instance in [9] an improved, lower-consumption Power Amplifier (PA) targeted for LTE applications is presented.

In contrast to the abundance of theoretical and simulation works there is a distinct lack of publications examining the practical aspects of power-saving. There is low amount of data about devices' actual power consumption, and implementation of "green" modes. One of the works that tries to quantify energy consumption of wireless networks based (partially) on measurements is [5]. There, in-depth review of power consumption of different types of BS is presented, together with mapping of the radiated RF power to the supply power of those devices. In another study [6], the focus is applying energy-adaptation solutions in function of the signal load in femto and pico cells. In contrast to that work, we don't believe that overcomplicated and state-of-the-art component/subsystem solutions will find quick application in femto and pico cells, where cost is a key factor. Our understanding is that it is much more important to grasp the capabilities and the limitations of currently existing hardware, since our research shows that significant power-savings are possible even in nowadays products with relatively minor, or at least trivial, modifications.

\section{EnABling Power-SAVE Mode ON FEMto}

\section{A. Femto Architecture And Consumption}

A typical generic architecture of a $3 \mathrm{G}$ femto $\mathrm{BS}$ (a femtocell, or just femto) is shown on Figure 1. Most of the 
elements can be found in any embedded system. In particular the architecture is quite close to that of a Wi-Fi Access Point.

The heart of the device is the Baseband processor, which implements most of the required HW functionality. It contains a generic application CPU core (e.g. ARM, PowerPC), a 3GPP modem, optimized co-processors or HW accelerators, crypto engine, and peripherals to support HSPA WCDMA functionality. The important (from power-consumption point of view) parts of the RF subsystem are: an ADCs+DACs IC (also often referred to as mixed signal frontend), a transceiver (Trx), pre-amplifier (also called Gain Block or Gain Amplifier - GA), and a power amplifier (PA).

A $3 \mathrm{G}$ femto also requires a stable master clock - usually a combination of a TCXO (temperature-compensated crystal oscillator) and a heater that keeps the TCXO at a stable temperature in order to eliminate clock drift caused by temperature change. Minimizing clock drift is required in order to meet the stringent clock stability requirements in UMTS systems.

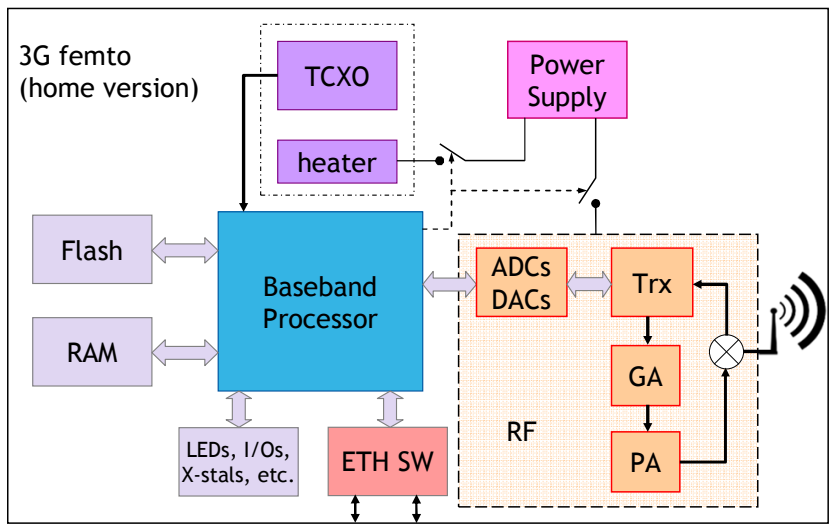

Figure 1. $3 \mathrm{G}$ femto block diagram

The rest of the $3 \mathrm{G}$ femto components will not be discussed in detail - these are Ethernet switch controller, Power supply subsystem (DC/DC converters), Flash and RAM, LEDs, switches, I/O interfaces, other crystal oscillators, logic ICs, etc.

The power consumption of a typical modern $3 \mathrm{G}$ femto is about 8-14 Watts. The figure does not include the external power supply efficiency (usually between $80 \%$ and $90 \%$ ). The power losses in the external power supply (usually a $220 / 110 \mathrm{~V}$ $\mathrm{AC}$ to $12 \mathrm{~V} \mathrm{DC}$ unit) will not be considered in the analysis below. Since losses in internal DC/DC converters (ranging from $5 \%$ to $10 \%$ ) are proportional to the load, those losses will be implicitly added to components/subsystems consumption figures. The consumption split per component/subsystem (in $\%$ ) is shown in Table I. Some of the data there comes directly from our measurements (the consumption of: the whole system, the radio, and the oscillator heater), while the rest is taken from the datasheets of the HW components. As seen in the table, Radio as a whole consumes the most power (almost half of the total), followed by the baseband processor $(25 \%)$, the Ethernet switch (15\%), and the TCXO heater (9\%).
TABLE I. KEY COMPONENTS POWER CONSUMPTION

\begin{tabular}{|c|c|c|}
\hline $\begin{array}{l}\text { Component / } \\
\text { Subsystem }\end{array}$ & $\begin{array}{c}\text { Power } \\
\text { share, \% }\end{array}$ & $\begin{array}{c}\text { RF parts } \\
\text { power } \\
\text { share, } \%\end{array}$ \\
\hline BB processor & 25 & \\
\hline ETH switch & 15 & \\
\hline MEM Avg. & 5 & \\
\hline Radio (incl. DC/DC) & 45 & \\
\hline $\mathrm{ADC} / \mathrm{DAC}$ & & 20 \\
\hline $\operatorname{Tr} x$ & & 25 \\
\hline PreAmp (GA) & & 20 \\
\hline PA & & 30 \\
\hline Other & & 5 \\
\hline Osc. Heater Avg. & 9 & \\
\hline Other & 1 & \\
\hline
\end{tabular}

An important observation from our experiments is that in the case of femto BS the ERP (Effective Radiated Power) is so low (in the order of $10 \mathrm{~s}$ of $\mathrm{mW}$ ), that variations of that power practically do not influence the electrical power consumption of the device. In other words as soon as the femto RF subsystem is powered on, its electrical consumption will be relatively stable in time, regardless of the variations of emitted RF power and cell load. This is confirmed in [5] as well. Thus, any approach to save electrical power just by manipulating the $\mathrm{RF}$ power will not be effective.

Another note is that the TCXO heater consumption is given for the case the TCXO has reached a stable temperature, in which case the heater only compensates for heat losses. The latter depend (among other factors) on the ambient air temperature and the heater temperature set point.

\section{B. Energy-Saving Mode Design}

When a femto is not being used (i.e. there are no active users), it can reduce its power consumption by going into a power-saving mode. In that mode different subsystems or components of the femto can be switched off or be put in a power-saving mode (when the component supports it). Each component brings a cost (or penalty) and benefit (or gain) to the system's power-save mode. The cost can be the time that is added to the wake-up delay of the femto, or how important/necessary it is the component to still remain switched on in a particular power-saving mode. In reality the cost also includes the redesign effort (in particular the one for changing the firmware) so that the switching off/on of the component in question is supported, but this aspect will not be considered in detail in the current work. The benefit is the relative amount (percentage) of power that can be saved by switching off a component.

According to their gain/cost ratio components/subsystems can be classified as: 
1) Good candidates to be switched off in power-saving modes -items with high gain/cost ratio:

- Radio: high power savings (45\%), low wakeup delay $(<0.5 \mathrm{~s})$.

- TCXO heater: medium power savings (9\% in steady state), negligible cost: does not influence the wakeup delay of the femto, and has no short-term importance.

2) Average candidates to be switched off in power-saving modes -items with medium gain/cost ratio:

- Baseband processor: high power savings (25\%), but high costs as well: considerable ( $>10$ s) wakeup delay due to power-up sequences and context restoring; huge software redesign effort (available firmware is typically written with no power-saving in mind).

- ETH switch: high power savings (15\%), but high cost: it is often necessary to maintain backhaul connectivity for a) keeping link with the $3 \mathrm{G}$ core, and for b) waking-up the femto once it is in a standby mode.

3) Bad candidates to be switched off in power-saving modes -items with low gain/cost ratio:

- Memory: medium-to-low power savings (5\%), high cost: Flash is always necessary when $\mathrm{BB}$ processor is on, and RAM is necessary for all modes except off.

- Logic ICs and oscillators: very low power-savings $(<2 \%)$, medium to high cost.

- $D C / D C$ converters: very low power-savings (low quiescent current), high cost (most of the time used).

Based on the above classification, and on estimation and experimentation, we determined that a good balance between granularity and complexity/effort would be to define four distinct operational (power) modes. Those modes are described below, ordered by "depth". The deeper a sleep mode is the more power is saved, but the more the cost of that mode is.

- On (or Active): The femto is in full operation, and is consuming maximum power.

- Standby: The femto is in "light" sleep and can wake-up quickly. The RF and the TCXO heater are switched off. Rx-related parts of the RF can be kept "on" if retaining reception capabilities is needed in this mode, but then the power-saving gains will be lower.

- Sleep: The femto is in "deep" sleep and might need considerable time to wake up due to initialization of additional components that were powered off. In this mode the only components that need to stay on are the power supply, and the generic CPU core in the baseband processor. Parts that can be in power-saving mode are the RAM and the ETH switch controller.

- Off: The femto is off and consumes no power. Waking up can take a significant amount of time, since the femto has to boot up, calibrate, and initialize itself.

Further, we have determined the characteristics of the power-saving modes, which are summarized in Table II, along with femto components/subsystems state in each mode.
TABLE II. POWER-SAVE MODES PROPERTIES

\begin{tabular}{|c|c|c|c|c|c|}
\hline & \multicolumn{4}{|c|}{ Power-Save mode } \\
\hline & & Active & Standby & Sleep & Off \\
\hline \multicolumn{2}{|c|}{ Wakeup time, s } & NA & 0.5 & $<10$ & $90-130$ \\
\hline \multicolumn{2}{|c|}{$\begin{array}{l}\text { Total power } \\
\text { consumption, } \%\end{array}$} & 100 & $45-66$ & $<20$ & 0 \\
\hline \multirow{12}{*}{ 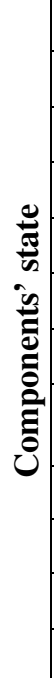 } & BB proc. & $\mathbf{X}$ & $\mathbf{X}$ & S & $\mathbf{O}$ \\
\hline & ETH switch & $\mathbf{X}$ & $\mathbf{X}$ & $\mathrm{M} / \mathrm{S}$ & $\mathbf{O}$ \\
\hline & RAM & $\mathbf{X}$ & $\mathbf{X}$ & S & $\mathbf{O}$ \\
\hline & Flash & $\mathbf{X}$ & $\mathbf{X}$ & $\mathbf{O}$ & $\mathbf{O}$ \\
\hline & Radio (supply) & $\mathbf{X}$ & M & O & $\mathbf{O}$ \\
\hline & ADC/DAC & $\mathbf{X}$ & M & $\mathbf{O}$ & $\mathbf{O}$ \\
\hline & $\operatorname{Trx}$ & $\mathbf{X}$ & $\mathbf{M}$ & $\mathbf{O}$ & $\mathbf{O}$ \\
\hline & $\begin{array}{l}\text { PreAmp } \\
(\mathrm{GA})\end{array}$ & $\mathbf{X}$ & $\mathbf{O}$ & $\mathbf{O}$ & $\mathbf{O}$ \\
\hline & PA & $\mathbf{X}$ & $\mathbf{O}$ & O & $\mathbf{O}$ \\
\hline & Other & $\mathbf{X}$ & M & $\mathbf{O}$ & $\mathbf{O}$ \\
\hline & TCXO Heater & $\mathbf{X}$ & $\mathbf{O}$ & $\mathbf{O}$ & $\mathbf{O}$ \\
\hline & Other & $\mathbf{X}$ & $\mathbf{X}$ & $\mathbf{X}$ & $\mathbf{O}$ \\
\hline
\end{tabular}

Legend: Components can be in state:

$$
\begin{aligned}
& \mathrm{X}-\text { on } \\
& \mathrm{O} \text { - off } \\
& \mathrm{M} \text { - optionally off } \\
& \mathrm{S} \text { - power-save }
\end{aligned}
$$

\section{Cell wake-up procedure}

During wake-up the dormant cell exits the power-saving mode and recovers to full-operational Active state.

If the challenges of the energy-saving mode design reside in the selection of circuits to switch-off in order to balance energy gains with system reactivity, the challenges of the wake-up design are mainly operational. The wake-up solutions differentiate by the way they perform two core operations proximity detection and wake-up execution. The first is to determine if an authorized UE is close to the dormant femtocell and needs connectivity. The second is how the wake-up decision is communicated to the dormant cell.

The simplest wake-up solutions are based on a static time programming of active-standby periods. The static approach can be used only when the period of usage is known or can be easily learned (e.g. opening hours of a commercial mall). Since the wake-up time is not critical, the energy gains during standby periods are optimal: near full switch-off can be used.

If the time distribution of active-standby periods is not known, dynamic wake-up must be used: the dormant cell is reactivated as soon as an authorized UE is detected nearby. The transition to active mode must be rapid enough to ensure wireless connectivity to the UE within an acceptable delay (few seconds).

Dynamic solutions can be classified into femto-based, UEbased, and NW-based. In the first one the femto performs 
proximity detection, usually by using a RF sniffer or by regularly turning on its radio to capture nearby UEs, if any. The wake-up command is triggered and executed internally.

In the UE-based solution the UE detects proximity and sends a wake-up message to the dormant cell. Proximity can be detected by sensing the presence of an alternative (secondary) coverage network, such as specific macro-cells, or a local network (Wi-Fi, etc...). The secondary coverage network is also used to send the wake-up command to the dormant cell.

In the NW-based case the mobile network (e.g. ANDSF server [10]) detects proximity and requests wake-up via the backhauling network. Proximity is detected by triangulation between (macro) cells or simply (but less precisely) by identifying the macro-cell which the mobile terminal is in. Furthermore, the mobile network can be assisted by the UEs, thanks to measurement reports. NW-based solutions require a standardized interface between macro and femto layer.

Each solution has specific advantages and drawbacks that make it more adapted to particular deployment case. For example, NW-based solutions would not be suitable when the femtocell is deployed in a (macro layer) coverage hole. Since no single solution would be practical and sufficient in all the possible deployment scenarios, we expect that commercial solutions will combine multiple wake-up techniques to be able to cover the majority of cases.

\section{PROTOTYPE IMPLEMENTATION}

We implemented an experimental prototype by enhancing a commercial $3 \mathrm{G}$ femtocell with a novel energy-saving mode and associated wake-up mechanism.

Our goal was to investigate several practical aspects of dynamic active-standby solutions, such as the effective energy savings and corresponding wake-up delays. Fully operational wake-up was also a major target, to validate protocol exchanges and system level behavior.

We implemented a standby mode (as defined in section III) to obtain reasonable energy savings with acceptable wake-up delays. When in standby mode the TCXO heater and the full radio subsystem (i.e. ADC/DAC, Trx, PreAmp, PA...) are turned off.

The choice of the wake-up solution has been driven by the need to limit the complexity and at the same time to preserve viability and practicability. Maximum energy savings (at system level) were not a main goal. We chose a dynamic UEbased wake-up technique, where detection and wake-up channel are provided by a secondary radio, $\mathrm{Wi}-\mathrm{Fi}$ in our case. This choice can be justified by the following points. It is independent from overlay macro layer, and works even if macro coverage is not available. Wi-Fi technology is currently everywhere - all recent smartphones have it, and commercial femtocells are progressively integrating $\mathrm{Wi}-\mathrm{Fi}$ modules. Finally, Wi-Fi and 3G (femto) have equivalent coverage areas, so proximity detection can be achieved with good precision.

Since our femto did not include a Wi-Fi module, we added an external Wi-Fi Access Point linked to the 3G femtocell via Ethernet connection, as shown in Figure 2.

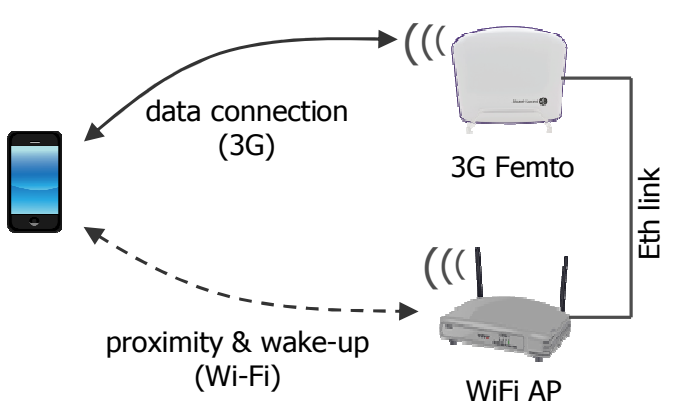

Figure 2. Prototype set-up

Clearly, this solution is not optimal from energy efficiency point of view, but is a good compromise between complexity and feasibility, and allows measurement flexibility.

When the femtocell is active, the Wi-Fi AP can be turnedoff (for energy savings) or kept on (for maximum capacity and data offload). Power consumption figures for the two modes are reported in the next section.

The femtocell remains active as long as there are UEs registered with it. When the last UE de-registers (due to switch-off or hand-over) the femtocell enters standby mode and notifies the Wi-Fi AP through the Ethernet link.

The Wi-Fi AP used for the prototype is a Broadcom BCM95354GR running OpenWrt, and its behavior has been modified as follows. The AP broadcasts a special SSID. UE authentication based on Probe Requests is enabled. The reception of a Probe Request triggers sending of a wake-up request to the femtocell via an Ethernet link. The emission of probe response is synchronized with the reception of wake-up confirmation from the femtocell.

On UE side, we used a dual-mode (3G+WiFi) HTC HD2, with Android 2.3.7. The UE has been programmed to periodically scan Wi-Fi networks for the special SSID, turn-on the $3 \mathrm{G}$ radio interface on reception of Probe Response, and finally turn-off the Wi-Fi interface on connection to $3 \mathrm{G}$ network. Note that UE Wi-Fi interface must be on only during the process of waking up the $3 \mathrm{G}$ femto - for the rest of the time the UE Wi-Fi module is mostly off to save energy.

\section{EXPERIMENTAL RESULTS}

We ran multiple active-standby cycles (50) and averaged (and normalized) measurements results for power consumption and state transition delays. The deviation of all values (except for some mentioned below) was within $10 \%$ of the average.

\section{A. Power consumption}

We report in Table III the measured power consumption for the entire system, as well for the $\mathrm{Wi}-\mathrm{Fi} \mathrm{AP}$ and the femtocell alone - in its different states (active and standby). Power consumed in standby mode $(4.5 \mathrm{~W})$ is reduced with about $55 \%$ compared to active state $(10 \mathrm{~W})$.

At system level, we report the overall power consumption in three different working conditions: femto standby, femto 
active, and capacity boost (Femto $+\mathrm{Wi}-\mathrm{Fi}$ ). We compare them to the consumption of a regular $3 \mathrm{G}$ femto (last row).

Despite the fact that the implemented solution is not energy optimal, savings are still achievable in Femto standby state (around 30\%). This is due to the fact that today's Wi-Fi AP consumes only a fraction of power consumed by a $3 \mathrm{G}$ Femto.

TABLE III. MEASURED POWER CONSUMPTION

\begin{tabular}{|c|c|c|c|}
\hline \multirow{2}{*}{$\begin{array}{c}\text { Working } \\
\text { conditions }\end{array}$} & \multicolumn{2}{|c|}{$\begin{array}{c}\text { Module power-save mode, } \\
\text { and consumption in W }\end{array}$} & $\begin{array}{c}\text { Total Power } \\
\text { consumption, W }\end{array}$ \\
\cline { 2 - 3 } & Femto & Wi-Fi AP & \\
\hline $\begin{array}{c}\text { Femto } \\
\text { standby }\end{array}$ & $\begin{array}{c}\text { Standby, } \\
4.5\end{array}$ & $\begin{array}{c}\text { On, } \\
2.5\end{array}$ & 7 \\
\hline $\begin{array}{c}\text { Femto } \\
\text { active }\end{array}$ & $\begin{array}{c}\text { On, } \\
10\end{array}$ & $\begin{array}{c}\text { Standby/Off, } \\
0.5\end{array}$ & 10.5 \\
\hline $\begin{array}{c}\text { Capacity } \\
\text { boost }\end{array}$ & $\begin{array}{c}\text { On, } \\
10\end{array}$ & $\begin{array}{c}\text { On, } \\
2.5\end{array}$ & 12.5 \\
\hline $\begin{array}{c}\text { Regular } \\
\text { femto }\end{array}$ & $\begin{array}{c}\text { On, } \\
10\end{array}$ & $/$ & 10 \\
\hline
\end{tabular}

\section{B. State transition delays}

We focused our measurements on the wake-up phase, decomposed in three delays of interest. $\Delta_{\text {detect }}$ is the time needed by the UE to detect the special Wi-Fi SSID (beacon) on its Wi-Fi interface and to send the Probe Request message. $\Delta_{\text {wakeup }}$ is the wake-up delay, during which the femto is reactivated. It is measured from the reception of the wake-up request to full operation of the $3 \mathrm{G}$ femtocell. $\Delta_{3 \text { gconnect }}$ is the additional time required to turn-on the UE $3 \mathrm{G}$ interface and to establish the $3 \mathrm{G}$ connection between the femtocell and the UE. The measured delays for different power-saving modes are reported in Table IV. Here the deviation of $\Delta_{\text {detect }}$ and $\Delta_{3 \text { gconnect }}$ reached $30 \%$ of the average, but this is independent of our implementation. Note that although "sleep" mode was not fully implemented, preliminary experiments allowed us to determine $\Delta_{\text {wakeup }}$ with a good precision.

TABLE IV. MEASURED StATE TRANSITION DELAYS (AVERAGE)

\begin{tabular}{|l|c|c|c|c|}
\hline \multirow{2}{*}{ Phase } & \multirow{2}{*}{ Symbol } & \multicolumn{3}{|c|}{ Time, s } \\
\cline { 3 - 5 } & & standby & sleep & off \\
\hline Proximity detection & $\Delta_{\text {detect }}$ & 2 & 2 & 0 \\
\hline Cell wake-up & $\Delta_{\text {wakeup }}$ & 0.5 & 10 & 120 \\
\hline $\begin{array}{l}\text { 3G connection } \\
\text { establishment }\end{array}$ & $\Delta_{\text {3gconnect }}$ & 6 & 6 & 6 \\
\hline
\end{tabular}

As expected, the femtocell wake-up from standby state $\left(\Delta_{\text {wakeup }}\right)$ is reasonably short $(0.5$ seconds), so dynamic wake-up can be done with acceptable delay. Using deeper saving mode (e.g. sleep mode) would increase largely the wake-up delay.

$\Delta_{\text {detect }}$ and $\Delta_{3 \text { gconnect }}$ correspond to the nominal execution time of standard $\mathrm{Wi}-\mathrm{Fi}$ and $3 \mathrm{G}$ procedures (scanning, association, connection, etc.). The measured delays are in line with the literature [10]. $\Delta_{\text {detect }}$ and $\Delta_{3 \text { gconnect }}$ are not impacted by the standby-wakeup mechanism, but can vary between different UEs, as they depend on proprietary implementations.

\section{CONCLUSIONS}

Cell stand-by is one of the most promising techniques today to reduce energy consumption of cellular base stations. The majority of existing studies assume that cells can simply enter standby mode - very few measurements and practical studies exist. In this paper we presented the results from a practical implementation of standby mode on a commercial 3G Femto. We showed that the choice of internal circuits to turn-off in standby mode is crucial to balance energy consumption reduction and wake-up delay. Savings can be in the order of $33-55 \%$ while still ensuring rapid wake-up $(<1 \mathrm{sec})$. Savings up to $80 \%$ can be achieved (sleep mode) if a wake-up delay of several seconds is acceptable. Higher savings come at price of much longer wake-up time. We measured state transition delays by implementing a simple wake-up procedure, based on a secondary $\mathrm{Wi}-\mathrm{Fi}$ radio used to detect UE proximity. This choice is not optimal from energy efficiency point of view but is feasible to implement. The measured cell wake-up delay is reasonably short $(0.5 \mathrm{sec})$, confirming that an attentive standby design allows the use of dynamic wake-up techniques. In the future we will implement other wake-up techniques and will compare the performance in terms of energy savings and delay.

\section{ACKNOWLEDGMENT}

The research leading to these results has received funding from the European Union Seventh Framework Programme (FP7/2007-2013) under grant agreement n. 257740 (Network of Excellence "TREND").

\section{REFERENCES}

[1] L.M. Correia et al., "Challenges and enabling technologies for energy aware mobile radio networks, "Communication Magazine, IEEE, vol.48, no. 11 , pp.66-72, November 2010

[2] W. Vereecken et al., "Evaluation of the potential for energy saving in macrocell and femtocell networks using a heuristic introducing sleep modes in base stations," EURASIP Journal on Wireless Communications and Networking 2012, 2012:170

[3] W. Vereecken et al., "The effect of variable wake up time on the utilization of sleep modes in femtocell mobile access networks," Wireless On-demand Network Systems and Services (WONS), 2012 9th Annual Conference on , pp.63-66, 9-11 Jan. 2012

[4] 3GPP Technical Specification TS 25.104, "Basestation (BS) radio transmission and reception (FDD)", http://www.3gpp.org/specifications

[5] G. Auer et al., "How much energy is needed to run a wireless network?," Wireless Communications, IEEE, vol.18, no.5, pp.40-49, October 2011

[6] B. Debaillie et al., "Opportunities for energy savings in pico/femto-cell base-stations," Future Network \& Mobile Summit (FutureNetw), 2011, pp.1-8, 15-17 June 2011

[7] L. Chiaraviglio, D. Ciullo, M. Meo, M.A. Marsan; , "Energy-efficient management of UMTS access networks," Teletraffic Congress, 2009. ITC 21 2009. 21st International, pp.1-8, 15-17 Sept. 2009

[8] A. Conte et al., "Cell Wilting and Blossoming for Energy Efficiency," Wireless Communications, IEEE, Vol. 18, No. 5, pp. 50-57, October 2011

[9] G. Watkins, J. Zhou, K. Morris, ">41\% efficient 10W envelope modulated LTE downlink power amplifier," European Microwave Integrated Circuits Conference (EuMIC), 2011, pp.260-263, 10-11 Oct. 2011

[10] M. C. Chuah, Q. Zhang, "Design and performance of $3 \mathrm{G}$ wireless networks and wireless LANs," Springer Science \& Business, 2006

[11] 3GPP TS 23.402 "Architecture enhancements for non-3GPP accesses", http://www.3gpp.org/specifications 